Nat Methods. 2019 May ; 16(5): 413-416. doi:10.1038/s41592-019-0368-0.

\title{
High-throughput identification of dominant negative polypeptides in yeast
}

\author{
Michael W. Dorrity ${ }^{1}$, Christine Queitsch ${ }^{1}$, and Stanley Fields ${ }^{1,2}$ \\ ${ }^{1}$ Department of Genome Sciences, University of Washington, Seattle, WA 98195 \\ ${ }^{2}$ Department of Medicine, University of Washington, Seattle, WA 98195
}

\begin{abstract}
Dominant negative polypeptides can inhibit protein function by binding to the wild type version or by titrating a ligand. Here, we use high-throughput sequencing of libraries composed of fragments of yeast genes to identify dominant negative polypeptides based on their depletion during cell growth. The method can uncover numerous inhibitory polypeptides for a protein and thereby define these fragments with exquisite resolution, even pinpointing individual residues with critical functional roles.
\end{abstract}

Dominant negative mutants disrupt the activity of a wild-type protein present in the same cell, predominantly by retaining interaction with another molecule while lacking a critical activity. ${ }^{1}$ For many proteins, a dominant negative polypeptide binds to a wild-type subunit to form an inactive mixed oligomer. In other cases, a dominant negative polypeptide titrates out an interacting protein, DNA site or small molecule. Dominant negative inhibition provides unique advantages. Unlike RNAi, which requires degradation of the targeted protein for its effect, dominant negative polypeptides can act almost immediately. Unlike CRISPR approaches that permanently alter a gene, dominant negative polypeptides can be conditionally and variably induced, ${ }^{1}$ features useful for inhibiting essential genes. Furthermore, because these polypeptides bind proteins based on structural features, not nucleic acid sequence specificity, they can inhibit individual functions of a protein or multiple proteins possessing a common domain. Despite their utility, dominant negatives are typically discovered only in the context of broader mutant screens. Larger-scale attempts in the yeast Saccharomyces cerevisiae identified polypeptide fragments that are toxic when overexpressed, but these studies were limited in sensitivity and capacity to track fragments. $2-5$

Users may view, print, copy, and download text and data-mine the content in such documents, for the purposes of academic research, subject always to the full Conditions of use:http://www.nature.com/authors/editorial_policies/license.html\#terms

Correspondence to Stanley Fields, fields@uw.edu, tel 206 616-4522.

Author contributions: M.W.D., C.Q., and S.F. conceived and interpreted experiments. M.W.D., C.Q., and S.F. wrote the manuscript. M.W.D. conducted experiments and data analysis.

Competing interests: The authors declare no competing interests.

Data Availability: High-throughput sequencing reads have been submitted to NCBI SRA under accession numbers SRR8573191 SRR8573214. 
We used high-throughput sequencing of DNA libraries encoding $S$. cerevisiae polypeptides to identify thousands of dominant negative gene fragments by their decreased frequencies in cells after growth selection. We initially tested the method with individual proteins. Yeast cells require homodimers of Ura3 to synthesize pyrimidines and thus to grow in media lacking uracil. We fragmented the $U R A 3$ gene to $\sim 200 \mathrm{bp}$, and cloned $>7,000$ fragments into a low-copy vector with a galactose-inducible promoter, which should produce 25 - to 100 fold more of a Ura3 fragment than natively expressed full-length Ura3. ${ }^{6,7}$ Triplicate cultures transformed with this library were induced with galactose and grown 48 hours without uracil (Fig. 1A). We sequenced inserts to track the frequency of each fragment before and after selection, allowing us to calculate a depletion score for each fragment. Values ranged from highly depleted ( $>1$ standard deviation below the median score) to neutral (output frequency similar to or greater than the starting frequency), with few fragments depleting before induction and selection (Supplementary Fig. 1A).

While most (940/1318) in-frame fragments had little effect, 378 showed dominant negative activity. We aligned these to $U R A 3$, controlling for coverage biases (Methods), to establish a per-position depletion score. Depleted fragments and per-position depletion scores correlated $\left(r^{2}=0.94, r^{2}=0.9\right)$ across replicates (Supplementary Fig. 1B-E). The most depleted region encompasses amino acids 70-160 (Fig. 1B, Supplementary Fig. 1F), which contains the dimer interface (Fig. 1C) and active site; the most depleted residue, threonine 100 (Fig. 1B), makes a critical substrate contact. ${ }^{8}$ This region also contains the smallest inhibitory polypeptides (Supplementary Fig. 2). Tested individually, two fragments from this region reduced growth in media lacking uracil by $15-30 \%$, while an out-of-frame control had no effect (Fig. 1D). As the selection does not exclude the identification of depleted fragments with non-specific effects, such as forming toxic aggregates or disrupting membranes, we tested dominant negative fragments in media containing uracil, where Ura3 is not essential, and found no growth defects (Supplementary Fig. 3).

We carried out a similar experiment with the transcription factor Hsf1, which contains conserved domains for DNA-binding, homo-trimerization and, putatively, autoregulatory repression. Hsf1 activates genes required for basal growth at $30^{\circ} \mathrm{C}$ and the heat-shock response at $37^{\circ} \mathrm{C}$, and is essential at both temperatures. We generated a library of $>12,000$ fragments of $H S F 1$, transformed yeast, induced expression and grew cells at either $30^{\circ} \mathrm{C}$ or $37^{\circ} \mathrm{C}$. Most fragments showed no strong change in depletion across the selection at either temperature, but 237 in-frame fragments depleted at $30^{\circ} \mathrm{C}$ and 434 at $37^{\circ} \mathrm{C}$. These depleted sets largely overlapped (192/316), suggesting that most fragments act independent of temperature. Alignments of depleted and control sets reveal three regions with dominant negative effects (Fig. 2A), roughly corresponding to the conserved domains.

To validate these effects, we measured the growth of cells expressing dominant negatives. A fragment containing the DNA-binding and trimerization domains was the most deleterious, at $30^{\circ} \mathrm{C}$ and $37^{\circ} \mathrm{C}$ (Fig. 2B). A fragment containing the trimerization domain alone reduced growth much more at $37^{\circ} \mathrm{C}$ than at $30^{\circ} \mathrm{C}$ (Fig. $2 \mathrm{~B}$ ), consistent with previous data ${ }^{9}$ and the production of nonfunctional trimers. A fragment corresponding to the autoregulatory domain in human $\mathrm{Hsf} 1 \mathrm{had}$ no effect at $30^{\circ} \mathrm{C}$, but reduced growth at $37^{\circ} \mathrm{C}$ by $15 \%$ (Fig. 2B), suggesting that this domain has activity in $S$. cerevisiae, despite contrary assertions. ${ }^{10-12}$ 
Depletion score minima corresponded to functional regions, including in the DNA recognition helix, a linker implicated in promoting DNA binding ${ }^{13}$ and two heptad repeats (Fig. 2A,C). Thus, the dominant negatives revealed new features of the extensively-studied Hsf1, such as the autoregulatory domain in yeast.

To test this approach across many genes simultaneously, we used a library of $>172,000$ yeast cDNA fragments, imposing a growth selection to obtain depletion scores. Because cDNA represents native expression levels, the library is biased toward highly expressed genes. Of 6,713 annotated yeast genes, 3,311 had at least one in-frame fragment with adequate sequencing depth (Supplementary Fig. 4A-B). Of these 3,311, 1,505 had at least one strongly depleted in-frame fragment. We also analyzed frequencies over three time points to calculate a slope for each depleted fragment (Supplementary Fig. 4C, Supplementary Table 1).

We sought to identify regions enriched for dominant negative fragments. From the 20,000 most depleted in-frame fragments, we mapped these to genes and then filtered based on the number overlapping fragments to 157 genes (Methods, Supplementary Fig. 5A-B,

Supplementary Data 1). We ranked each gene by its potential to generate dominant negatives (Supplementary Fig. 6A, Supplementary Table 2). The final list of 157 (annotated in Supplemental Table 3) is primarily limited by the number of fragments tested per gene; 257 more genes can be recovered at a less stringent threshold (Supplementary Table 4). The set of 157 genes contains 114 assayed as full knock-outs. ${ }^{14}$ The distribution of fitness effects for these 114 largely overlapped the background distribution (Supplementary Fig. 6B). Thus, dominant negative fragments of many genes reduce yeast growth even though knock-outs do not, likely due in part to the fragments inhibiting multiple genes.

The 157 genes were enriched for components of the ribosome and translation, consistent with their essential nature and abundance. Eleven cytoplasmic and three mitochondrial ribosomal proteins contained regions with overlapping dominant negative fragments (Supplementary Fig. 7). As ribosomal proteins are monomeric, these dominant negatives are not interfering with homo-oligomerization and may instead inhibit ribosome assembly. Most cytoplasmic ribosomal proteins in yeast are encoded by highly similar paralog pairs; the eleven with dominant negatives include two with regions from both paralogs and two from only one. Thus, single dominant negative fragments can target two proteins with similar structures, and a fragment from a single protein in a complex can inhibit the activity of the complex. Seven of the cytoplasmic ribosomal proteins map onto the cryo-EM structure ${ }^{15}$ (Supplementary Fig. 8), showing regions sensitive to inhibition. We observed hits in other proteins necessary for translation, including the domain of tRNA synthetases that binds the anticodon (Fig. 3A, Supplementary Fig. 9), as shown previously for a synthetase fragment. ${ }^{16}$ We found dominant negative regions in genes for the cell cycle, amino acid metabolism, tRNA processing, stress response, cytoskeleton and cell wall maintenance, suggesting that this method is broadly applicable in targeting cellular processes.

To test for non-specific toxicity, we characterized fragments from three biosynthetic genes, $A D E 3$ (for adenine), THR1 (for threonine), and ARO1 (for aromatic amino acids). Null mutants in these genes show slight growth defects even in complete media. ${ }^{14}$ We showed 
that the Thr 1 fragment reduced growth by a further $17 \%$ in media lacking threonine, and the Ade3 fragment by a further $16 \%$ in media lacking adenine, whereas the Thr1 fragment had no effect in media lacking adenine nor the Ade 3 fragment in media lacking threonine (Supplementary Fig. 10A-D), suggesting specificity for targets. Fragments from Aro1, Ade3 and Thr1 reduced growth in media lacking phenylalanine, indicating that this media cannot provide evidence for specificity. Overall, these results suggest that non-specific toxicity is not a primary signal in the data.

To assess whether different environmental conditions reveal unique dominant negatives, we screened the cDNA library at $37^{\circ} \mathrm{C}$. The large majority (97.8\%) of dominant negative polypeptides that depleted in basal conditions also depleted at $37^{\circ} \mathrm{C}$ (Fig. 3B). We found 508 depleted only in heat shock, with the most strongly depleted corresponding to a region having temperature-specific activity (Fig. 3B, inset from Fig. 2A). A fragment derived from the phosphatase $\mathrm{Sac} 1(64-128)$ that showed greater depletion at $37^{\circ} \mathrm{C}$ than $30^{\circ} \mathrm{C}$ corresponds to the interface with the Vps64 protein (Fig. 3C). Heterozygous truncations of FIG4, the human homolog of $S A C 1$, are associated with familial amyotrophic lateral sclerosis, ${ }^{17}$ suggesting that dominant negative activity may contribute to this disease. That some fragments inhibited growth only at high temperature suggests that their mode of action is revealed under stress. We also found about twice as many fragments $(1,248)$ with strong dominant negative activity only at $30^{\circ} \mathrm{C}$; temperature may affect the availability of the target or the binding interaction itself.

The method developed here can be applied to specific genes or entire libraries from any organism with suitable genetic tools. The timing, magnitude, and spatial pattern of inhibitor activity can be controlled using inducible expression. The resulting data should allow the definition of functional domains corresponding to interaction surfaces, particularly mediating homo-oligomerization or protein-protein interaction, although the mere identification of a dominant negative polypeptide does not reveal its mechanism of action. Remarkably, the method can pinpoint residues corresponding to local minima, which may contact a ligand. In 3'-phosphoglycerate kinase (Pgk1), which has multiple minima distant in primary sequence but nearby in three-dimensional structure, the minima correspond to internal structural features rather than ligand interaction (Supplementary Fig. 11); the inhibition may instead relate to protein folding. The homology between many yeast and human proteins suggests that dominant negative fragments of mammalian proteins can be found. Given the diversity of selection schemes, this method might address such questions as condition-specific protein interactions, species incompatibilities arising from protein interactions in hybrid diploids, and fine-tuning of biological circuits at the protein level, in addition to providing specific, rapidly acting reagents for functional genomics.

\section{Methods}

\section{Fragment library construction and transformation.}

For individual gene libraries, the URA3 and $H S F 1$ genes were amplified via PCR, and fragmented to an average length of $200 \mathrm{bp}$ using Illumina Nextera transposition. Transposed fragments were amplified via PCR, purified using DNA Clean and Concentrator (Zymo Research) columns and cloned into a low copy, galactose-inducible expression vector by 
Gibson assembly. Because each fragment contained a 5' adaptor sequence (AGATGTGTATAAGAGACAG) and 3' adaptor sequence (CTGTCTCTTATACACATCT) resulting from the transposition, the vector was designed to translate through these sequences such that each in-frame fragment would be translated as MKDVYKRQ-NNNLSLIHILTD $^{* *}$; stop codons are present downstream of the 3' adaptor in all possible reading frames. To generate a cDNA-derived fragment library, RNA was isolated from yeast cells via acid-phenol extraction, and polyA-specific cDNA was synthesized and transposed to obtain gene fragments. Fragment sizes ranged from $20 \mathrm{bp}$ to $1793 \mathrm{bp}$; the median length was $200 \mathrm{bp}$, and half of the total fragments were between $138 \mathrm{bp}$ and $282 \mathrm{bp}$ in length.

Electromax (Thermo Fisher) E. coli cells were transformed with the library assemblies and used to amplify a large pool of plasmid for subsequent yeast transformation. Haploid yeast cells (W303 strain) were transformed with fragment libraries such that each cell received a low-copy plasmid carrying a TRP1 gene for plasmid selection; cells were therefore diploid only at the locus corresponding to the fragment it contains.

\section{Cell growth selections.}

Cells were maintained in log phase for all growth selections. For the URA3 experiment, yeast cells containing the $U R A 3$ fragment library were grown overnight without selection for Ura3 function in synthetic complete medium lacking tryptophan (SC-TRP, for plasmid selection only). After 2 hours of pre-induction with galactose, these cells were washed to remove excess media and transferred to induction media lacking tryptophan and uracil (SCTRP-URA, +galactose) for the selective growth condition. After reaching log phase, the cells were back-diluted and grown again in fresh SC-TRP-URA, +galactose for another round of growth selection. The $H S F 1$ experiment was conducted in a similar fashion, except that after the 2 hour pre-induction, the culture was split such that one population continued growth at $30^{\circ} \mathrm{C}$ (SC-TRP, +galactose), and the second population was shifted to $37^{\circ} \mathrm{C}$ (SCTRP, +galactose). Each culture was back-diluted during log phase to undergo additional growth selection. Growth selections for the cDNA experiment were conducted as for the $H S F 1$ experiment. In the growth selections, care was taken to transfer enough cells for at least a 1000-fold coverage of the initial fragment library size, which minimizes the effects of sampling error when passaging cultures.

\section{General toxicity growth experiments.}

For three biosynthetic genes identified in the cDNA screen, $A D E 3, T H R 1$, and $A R O 1$, we analyzed effects on overall cell growth compared to growth in conditions wherein each gene was essential. $A D E 3$ is essential for growth in media lacking adenine, THR1 is essential for growth in media lacking threonine, and $A R O 1$ is essential for cell growth in media lacking aromatic amino acids. We asked whether the effects of these fragments were magnified when the relevant nutrient was omitted by growing cells in the absence of threonine, adenine or phenylalanine.

\section{High-throughput sequencing and analysis of polypeptide fragments.}

Sequencing was carried out on Illumina's NextSeq platform. DNA sequencing libraries were prepared from plasmids extracted from yeast populations (Yeast Plasmid Miniprep II, Zymo 
Research) before and after selection, as well as from the initial plasmid input. These plasmids were used as template for PCR amplification ( $<15$ cycles) that added sequencing adaptors, as well as $8 \mathrm{bp}$ sample indexes. Paired-end reads spanning the fragment library were generated at a median of 1 million reads per sample for individual gene fragment libraries, and between 15-50 million reads per sample for the larger cDNA fragment libraries. The position and orientation of each fragment were determined by aligning each set of reads to the respective gene of origin, or to the full set of coding ORFs (SGD). Reads were aligned using Bowtie 2 . The frequency of each fragment was determined by counting unique fragments with identical gene of origin, DNA start site, and DNA stop site, and orientation (ex. HSF1_202_431_+). Each fragment was assigned a reading frame based on its start position. Fragments with less than five reads were filtered from analysis steps due to inadequate coverage.

Fragments were classified for the Ura3 and Hsf1 selections as depleted if they had a score greater than one standard deviation below the median per-fragment $\log 2$ depletion distribution. Dominant negative regions of individual gene selections for Ura3 and Hsf1 were determined by computing per-position fragment coverage within each gene and taking the ratio of in-frame depleted fragments to out-of-frame depleted fragments, each normalized by the coverage of equivalent numbers of fragments with neutral scores, i.e. a depletion score $\left(-\log 2\right.$ [coverage in-frame depleted/neutral $_{\text {/ coverage }}$ out-of-frame depleted/neutral]). Per-position coverages for each set of fragments were computed using the genomecov tool in Bedtools ${ }^{18}$. For Ura3 and Hsf1, any position with fewer than 25 fragments covering that position were excluded from analysis; this filter excluded positions on either end of the gene, where fragment coverage was lower.

For the cDNA library, we used the 20,000 most depleted in-frame fragments, along with equal numbers of out-of-frame and neutral fragments, and mapped these back to individual genes. Dominant negative regions of genes from the cDNA library were determined by (1) filtering out genes with fewer than 5 in-frame fragments; (2) filtering out genes with fewer than 4 overlapping in-frame fragments; and (3) filtering out genes whose number of overlapping in-frame fragments was less than 2-fold higher than the number of overlapping out-of-frame fragments. We filtered this set to genes with at least 5 in-frame fragments, identifying 456 genes (corresponding to 6,327 fragments). We filtered the 456 for genes with at least four overlapping fragments and with two times more in-frame depleted than in-frame neutral fragments, leaving 157 genes.

\section{Gene annotation in cDNA library.}

Dominant negative regions were annotated by SGD annotations. Genes corresponding to processes cited in the text include those for the cell cycle (PCL5, SRM1, RME1, CDC34); amino acid metabolism (LYS14, CDC34, HIS1, THR1, ADE3, ARO1, ARO8); tRNA processing (STP4, STP1, SRM1, SOL3, RPN2, SEN1); stress response (SSA1, TPS1, GPD1, SSA4, HSP12, SSC1, UTH1, SSA2, HSP60, SSE1); cytoskeleton (ARC15, LSB5, RVS167); and cell wall maintenance (CCW12, CWP2, UTH1). 


\section{Supplementary Material}

Refer to Web version on PubMed Central for supplementary material.

\section{Acknowledgements:}

We thank Robert Waterston and Evan Eichler for comments on the manuscript. The work was supported by NIH grant GM114166 to C.Q. and S.F. and NIH grant P41 GM103533 to S.F.

\section{References}

1. Herskowitz I Functional inactivation of genes by dominant negative mutations. Nature 329, 219-222 (1987). [PubMed: 2442619]

2. Akada R, Yamamoto J \& Yamashita I Screening and identification of yeast sequences that cause growth inhibition when overexpressed. Mol. Gen. Genet 254, 267-274 (1997). [PubMed: 9150260]

3. Ramer SW, Elledge SJ \& Davis RW Dominant genetics using a yeast genomic library under the control of a strong inducible promoter. Proc. Natl. Acad. Sci. U. S. A 89, 11589-93 (1992). [PubMed: 1454852]

4. Stevenson LF, Kennedy BK \& Harlow E A large-scale overexpression screen in Saccharomyces cerevisiae identifies previously uncharacterized cell cycle genes. Proc. Natl. Acad. Sci. U. S. A 98, 3946-3951 (2001). [PubMed: 11274415]

5 . Boyer J et al. Large-scale exploration of growth inhibition caused by overexpression of genomic fragments in Saccharomyces cerevisiae. Genome Biol 5, R72 (2004). [PubMed: 15345056]

6. Saldanha AJ, Brauer MJ, Botstein D Nutritional homeostasis in batch and steady-State culture of yeast. Mol. Biol. Cell 15, 4089-4104 (2004). [PubMed: 15240820]

7. Lim MK et al. Galactose induction of the GAL1 gene requires conditional degradation of the Mig2 repressor. Biochem. J 435, 641-649 (2011). [PubMed: 21323640]

8. Chan KK et al. Mechanism of the orotidine 5-monophosphate decarboxylase-catalyzed reaction: Evidence for substrate destabilization. Biochemistry 48, 5518-5531 (2009). [PubMed: 19435314]

9. Voellmy R Dominant-positive and dominant-negative heat shock factors. Methods 35, 199-207 (2005). [PubMed: 15649847]

10. Liu XD, Liu PC, Santoro N \& Thiele DJ Conservation of a stress response: human heat shock transcription factors functionally substitute for yeast HSF. EMBO J 16, 6466-6477 (1997). [PubMed: 9351828]

11. Rabindran SK et al. Regulation of heat shock factor trimer formation: Role of a conserved leucine zipper. Science 259, 230-234 (1993). [PubMed: 8421783]

12. Chen Y, Barlev N. a, Westergaard O \& Jakobsen BK Identification of the C-terminal activator domain in yeast heat shock factor: independent control of transient and sustained transcriptional activity. EMBO J 12, 5007-18 (1993). [PubMed: 8262043]

13. Flick KE, Gonzalez L, Harrison CJ \& Nelson HCM Yeast heat shock transcription factor contains a flexible linker between the DNA-binding and trimerization domains: Implications for DNA binding by trimeric proteins. J. Biol. Chem 269, 12475-12481 (1994). [PubMed: 8175654]

14. Yoshikawa K, Tanaka T, Ida Y, Furusawa C, Hirasawa T, and Shimizu H, Comprehensive phenotypic analysis of single-gene deletion and overexpression strains of Saccharomyces cerevisiae. Yeast 28, 349-361 (2011). [PubMed: 21341307]

15. Armache J-P et al. Cryo-EM structure and rRNA model of a translating eukaryotic $80 \mathrm{~S}$ ribosome at 5.5-A resolution. Proc. Natl. Acad. Sci. U. S. A 107, 19748-19753 (2010). [PubMed: 20980660]

16. Michaels JEA, Schimmel P, Shiba K \& Miller WT Dominant negative inhibition by fragments of a monomeric enzyme. Proc. Natl. Acad. Sci. U. S. A 93, 14452 (1996). [PubMed: 8962072]

17. Chow CY et al. Deleterious Variants of FIG4, a Phosphoinositide Phosphatase, in Patients with ALS. Am. J. Hum. Genet 84, 85-88 (2009). [PubMed: 19118816]

18. Neudegger T, Verghese J, Hayer-Hartl M, Hartl FU \& Bracher A Structure of human heat-shock transcription factor 1 in complex with DNA. Nat. Struc. \& Mol. Bio 23, 140 (2016). 
19. Quinlan AR BEDTools: the Swiss-army tool for genome feature analysis. Curr. Prot. in Bioinform 47, 11-12 (2014). 


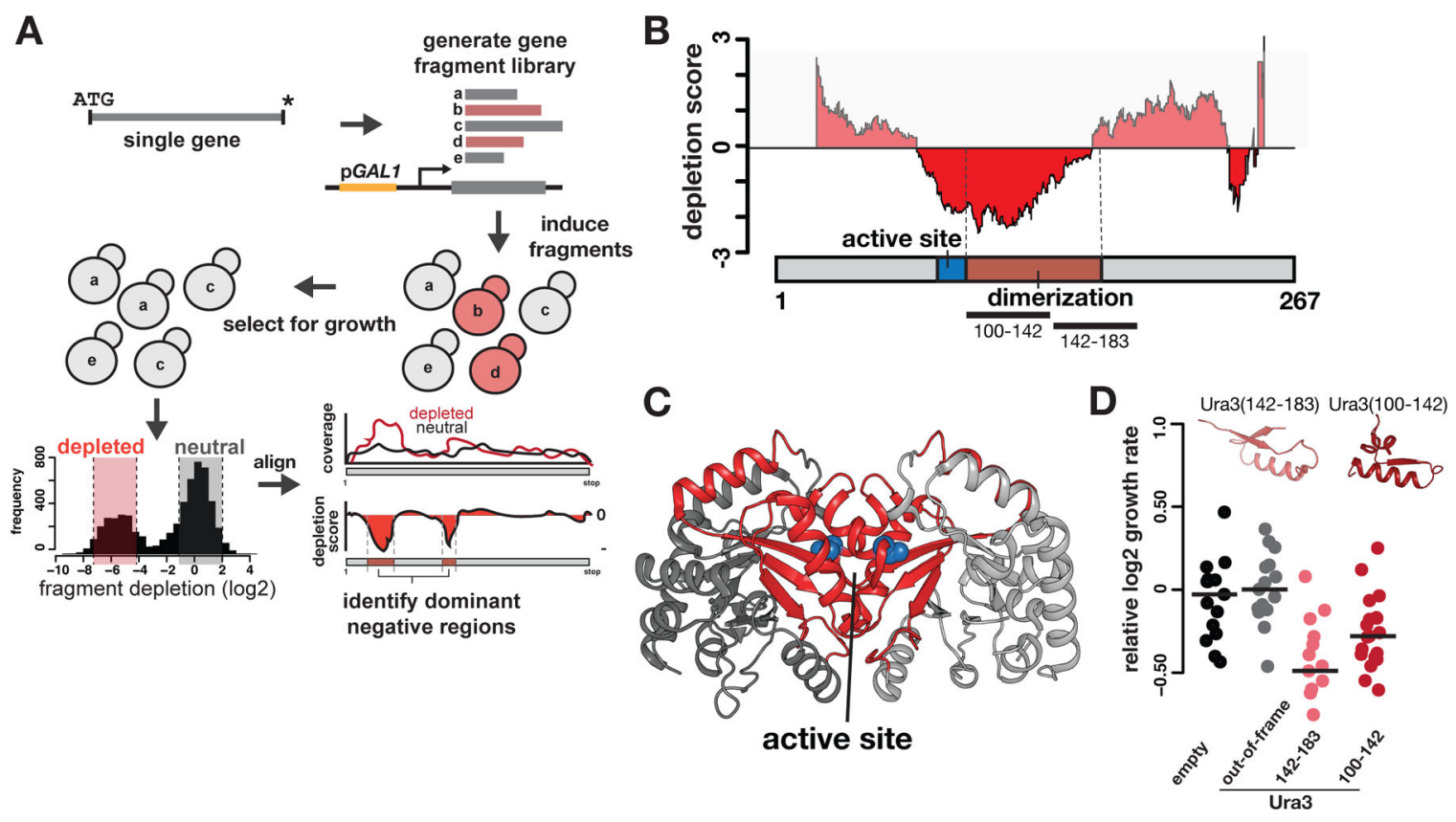

Figure 1. Dominant negative polypeptides can be identified in high-throughput and correspond to known protein domains.

(A) Schematic of the experimental and computational pipeline to identify dominant negative polypeptides in high-throughput. Briefly, each fragment is classified as depleted (putative dominant negative) or neutral based on its change in frequency in the output population, and the positional information of fragments from each of these classes is used to align them back to the URA3 gene. The pileup generated by alignment of dominant negative fragments is compared to the pileup generated from neutral fragments to generate a "depletion score" that is more negative in regions of the gene with an excess of dominant negative fragments (see Methods). (B) Negative depletion scores indicate regions with excess dominant negative fragments. Positions on either end of Ura3 with no computed depletion score had fewer than 25 mapped fragments. A model of the Ura3 protein is shown below, with active site and dimerization domain highlighted. (C) The dominant negative region identified in (B) was mapped onto the crystal structure of the Ura3 homodimer (PDB: 3gdk)8; this region covers nearly all residues in the homodimer interface. Threonine 100 is shown in blue in the indicated active site. (D) Barplots showing individual growth rates, measured in liquid culture using continuous measurements on a plate reader, for yeast cells containing a wildtype copy of the URA3 gene and one of three fragments (amino acids 10-43 out of frame, amino acids 100-142, and amino acids 142-183). Lines correspond to median values. The regions from the Ura3 structure corresponding to the two in-frame fragments are shown above the barplots. Growth rates are normalized to a strain growing without fragment expression. Both Ura3(142-183) and Ura3(100-142) show significant growth defects relative to the empty vector (one-sided $\mathrm{p}$-value $=0.0007$ and 0.00098 respectively, $\mathrm{n}$ $=15,15,15,19$ for each strain, in order of plot). 
A

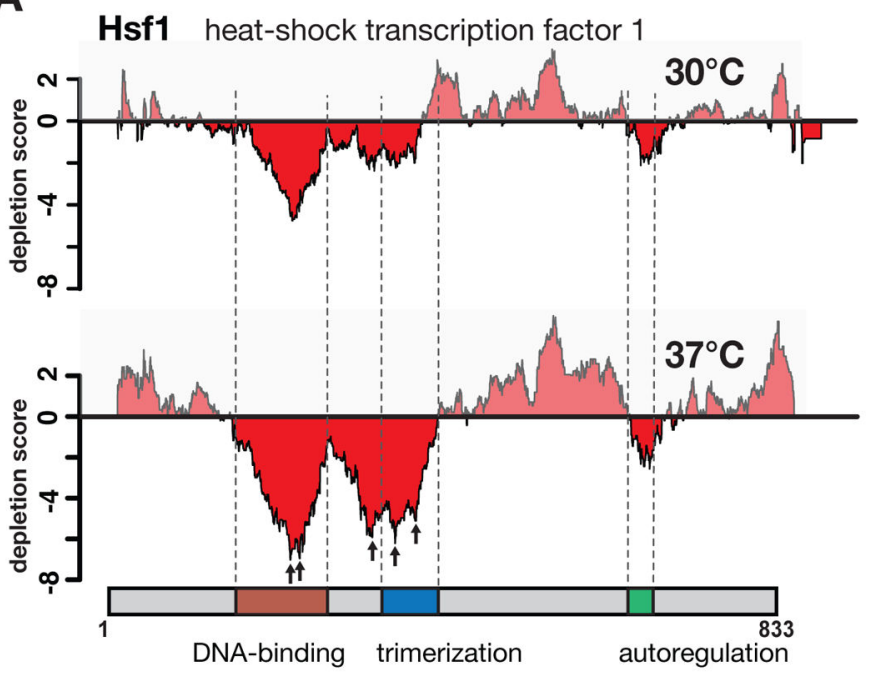

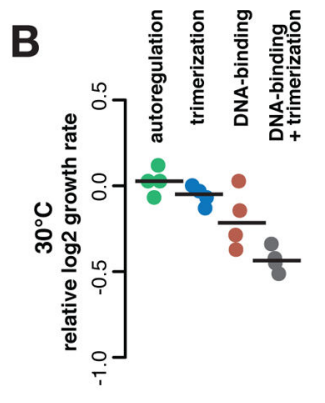

C

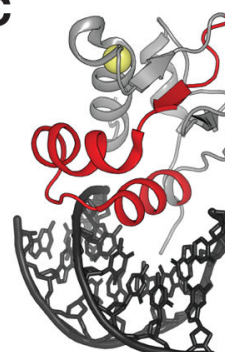

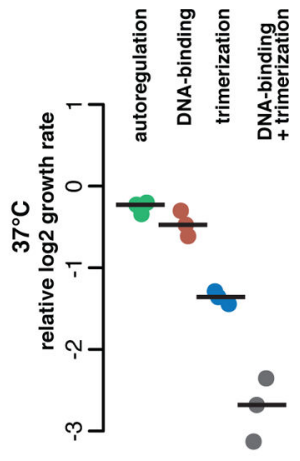

Figure 2. Identification of conditional dominant negative polypeptides in the essential heat-shock transcription factor Hsf1.

(A) Plots showing depletion scores as in Fig. 1B for dominant negative polypeptide identification in Hsf1. Depletion scores for cells grown at $30^{\circ} \mathrm{C}$ are shown in the top panel, and scores for cells grown at $37^{\circ} \mathrm{C}$ are shown in the lower panel. Local minima are indicated with arrows. (B) Individual growth rates measured by plate reader for the regions identified in (A). Growth rates for all fragment-containing strains are normalized to that of cells containing an empty vector. Lines correspond to median values; $n=3$ for all strains. (C) A region of 35 amino acids containing the local minima in the DNA-binding domain (arrows in A) is mapped onto the structure of the human Hsf1 DNA-binding domain (PDB: $5 \mathrm{~d} 5 \mathrm{v})^{18}$; spacer and trimerization minima do not appear in the protein structure. 


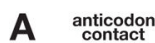

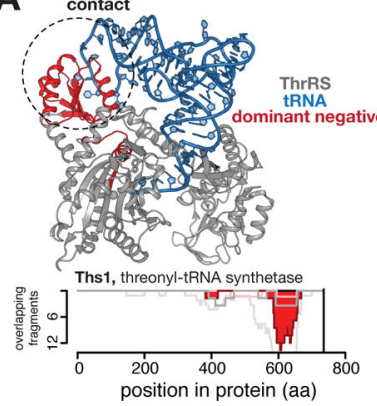

B

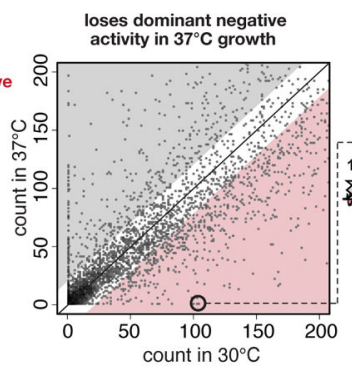

C

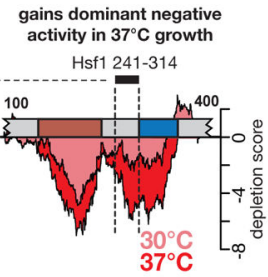

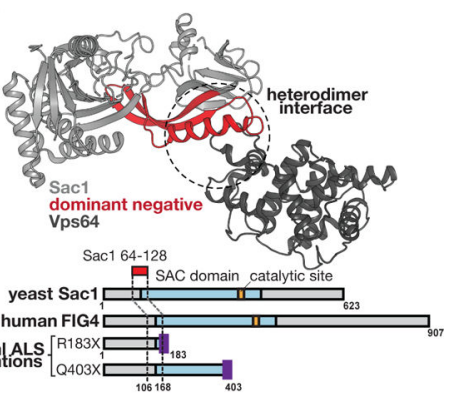

Figure 3. Dominant negative inhibitors of identified in a full-genome screen.

(A) The dominant negative region identified in the threonyl-tRNA synthetase (red) mapped onto the crystal structure (gray) in complex with its cognate tRNA (blue). The dominant negative domain (red) corresponds to the anticodon binding region of the tRNA synthetase. (B) The frequency of each fragment in $30^{\circ} \mathrm{C}$ (x-axis) and $37^{\circ} \mathrm{C}$ (y-axis) conditions. The fragment with the greatest dominant negative activity specific to $37^{\circ} \mathrm{C}$ derived from the linker region between the DNA-binding domain and trimerization domain of Hsf1 (inset from Fig. 2A). (C) A temperature-sensitive dominant negative fragment (red) derived from the yeast $S A C 1$ gene is highlighted at a heterodimer interface in the crystal structure of the Sac1-Vps64 complex. Below is a schematic representation of the yeast Sac1 protein and its human homolog FIG4. The SAC domain is shown in light blue, and the catalytic site in orange. Two truncation variants of human FIG4 associated with dominant ALS are shown below, and the conserved position of the dominant negative fragment identified in yeast is shown with dashed lines. 